\title{
An Optimal Current Control of Interlink Converter Using an Explicit Model Predictive Control
}

\author{
Ismi Rosyiana Fitri and Jung-Su Kim \\ Department of Electrical and Information Engineering, Seoul National University of Science and \\ Technology, Seoul, Korea
}

\section{]jfis}

\begin{abstract}
An interlink converter (IC) presents in a hybrid microgrid allowing a bidirectional power exchange between AC and DC sub-grids. In this paper, a model predictive control (MPC) -based IC current controller is employed, taking the current constraint into account to ensure the safety of IC. To reduce online computation burden of the MPC, a tabular form of MPC named Explicit MPC is applied. The simulation result shows that the proposed IC controller successfully maintains the power supply-demand balance of the hybrid microgrid. Moreover, the proposed method keeps the power exchange value in the acceptable range, particularly under high loading condition.
\end{abstract}

Keywords: Hybrid microgrid, Interlink converter, MPC

\section{Introduction}

Over the past century, generating clean energy has become an important issue due to the increasing awareness of the environmental issue. On the other hand, the number of renewable energy resources, such as wind turbine, photovoltaic and fuel cell, rapidly grow as the impact of science and technology. A microgrid is considered as a practical solution to integrate the renewable resources and provide the demand power. Microgrid is an energy system consisting of distributed energy sources and loads, and typically operates parallel with a power grid, although it can also work independently or synchronously with another microgrid.

Traditionally, microgrid consists of several AC distributed generators. Power converters are

used to supply DC loads and connect DC power sources. In the process of energy conversion, there would be power loss inevitably. As a consequence, the efficiency decreases as the increasing number of the power converter. Some studies consider a hybrid AC/DC microgrid to incorporate both $\mathrm{AC}$ and $\mathrm{DC}$ power sources [1, 2]. The hybrid AC/DC microgrid consists of two independent microgrids, AC and DC microgrid, which are linked by one or more interlink converter (IC). The necessary power conversion of AC/DC hybrid microgrid is less than the conventional one as the AC sub-grid mainly provides the AC load, and the DC sub-grid serves for the DC load primarily. The IC delivers power exchanged when one of sub-grid cannot manage its power supply-demand balance. For instance, when AC sub-grid experiences imbalance power, the IC transmits the necessary power from DC to AC-sub-grid.

In grid-connected mode, the utility grid is connected to the microgrid. The frequency stability of AC-sub-grid is guaranteed as the utility grid performs as the primary source. When 
a fault occurs in the utility grid, microgrid disconnects from the utility grid and changes its operating mode to the stand-alone mode. In this mode, the microgrid has to maintain the frequency, in AC sub-grid, and voltages, in both sub-grids. The IC takes an essential role as it provides a sufficient power exchange to maintain the power supply-demand balance of both sub-grids for any operating mode. In this regard, several control methods are studied, for instance, a droop-control is proposed by [1, 2] to determine the power flow from one sub-grid to the other. In [3], the coordination of IC and the battery controller is studied. Robust current control for the IC is investigated by [3]. Some literatures [4-7] show interest in the optimal control method for the IC controller. For example, an adaptive hysteresis control method is designed by [4] such that the switching loss of the traditional hysteresis is reduced. Another used a finite state model predictive control (MPC) to include the switching state in the optimization cost function [5]. While [6] shows that the linear quadratic regulator (LQR) method have superior performance compared to the proportional integrator (PI) controller. Also, an unconstrained MPC-based IC current controller is applied in [7]. In these literatures [1-3, 55-8], the control signal is saturated to prevent overmodulation in the signal modulator.

The focus of this paper is placed on employing an optimal control such that it regulates the output current while respecting the input constraint. A MPC has been widely used, for instance in [9, 10] to solve a control problem while satisfying a set of constraints. In this paper, a constrained MPC is considered to include the input constraint in the current controller. By doing that, the saturation function applied in $[1,-3,5,-8]$ can be eliminated. Furthermore, due to safety reason, the current output has to be limited in order to keep the power exchange below, or equals, to its maximum value. Conventionally, a saturation function is used in the reference value. Hence, under the assumption that the current controller yields a small tracking error, the power exchange is maintained in its acceptable value. However, this method is trivial, especially when the maximum power is required. Thus, this paper includes the state constraint in the design of MPC to avoid large current output. In order to consider constraints in the MPC, the controller has to solve the optimization problem every sampling time which may require huge computation burden. However, the IC hast fast sampling time. To this end, this paper employs an Explicit MPC to reduce online computation burden. The Explicit MPC solves the optimization problem offline and represents the optimal solution in a table-form. The simulation results demonstrate the proposed control successfully maintains the stability of

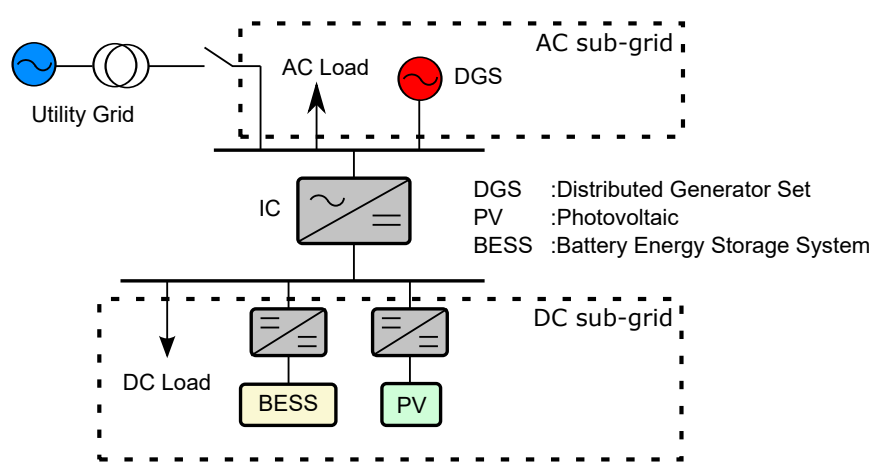

Figure 1. An AC/DC hybrid microgrid.

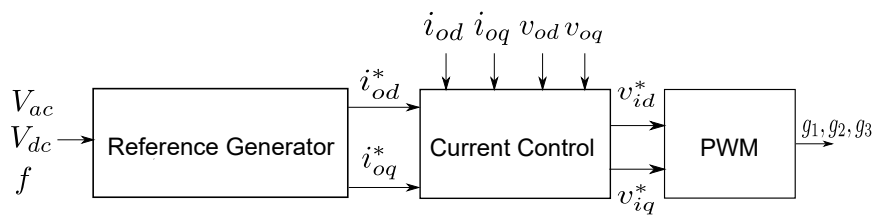

Figure 2. An AC/DC hybrid microgrid.

microgrid while preventing the power exchange from exceeding the maximum value.

\section{Hybrid Microgrid}

A hybrid microgrid is decomposed by two sub-grids: AC and DC sub-grids, as shown in Figure 1. The power sources and loads are placed in the sub-grid, depending on their inherent current type. An IC is used to incorporate both sub-grids and allows bidirectional power to flow from both the sub-grids alternately. When there is a power supply-demand imbalance in one sub-grid, the necessary power is transferred from the other sub-grid. With that in that mind, the IC establishes enough power exchange such that the power supply-demand balance is achieved in both sub-grids.

Figure 2 shows a typical block diagram in IC controller based on current-control mode. The controller mainly consists of three control blocks: a reference generator, a current controller, and pulse-width modulator (PWM). By denoting the IC current in $d q$-frame as $i_{o d}$ and $i_{o q}$, the power exchange is determined by imposing the IC current references $i_{o d}^{*}$ and $i_{o q}^{*}$ by the first control block as shown in Figure 2 In the $\mathrm{AC}$ sub-grid, the imbalance of active power affects the frequency stability. Meanwhile, it corresponds to the DC bus voltage deviation in the DC sub-grid. Since reactive power only exists in AC microgrid, the reactive power can only be transferred from $\mathrm{DC}$ to $\mathrm{AC}$ sub-grid. The reactive power imbalance decreases the $\mathrm{AC}$ voltage from 
the nominal value. These relations can be represented by the following equations

$$
\begin{aligned}
& f-f_{0}=k_{1}\left(P_{A C}-P_{A C, 0}\right), \\
& V_{A C}-V_{A C, 0}=k_{2}\left(Q-Q_{0}\right), \\
& V_{D C}-V_{D C, 0}=k_{3}\left(P_{D C}-P_{D C, 0}\right),
\end{aligned}
$$

where $P_{A C \text { or }(D C)}$ and $Q$ are the $\mathrm{AC}$ (or DC) active and reactive power, respectively, while $f$ and $V_{A C}$ or $(D C)$ denote the frequency and $\mathrm{AC}$ (or DC) voltage. The nominal value of $f$ is written as $f_{0}$, and similarly for other variables. The droop coefficients are represented by $k_{i}$.

The droop control for a hybrid microgrid is formulated by combining the sub-grids' droop characteristic. Intuitively, comparing the frequency and DC voltage deviations indicates which sub-grid has more severe active power imbalance. PI control is implemented to achieve equal power loading of the $\mathrm{AC}$ and DC sub-grids. The frequency and DC voltage are converted to per-unit (pu) values since both have a different unit base. Hence, the power exchange reference is given by:

$$
\begin{aligned}
& P_{I C}^{*}=\left(K_{p}+\frac{K_{i}}{s}\right)\left(c_{1} \Delta f_{p u}-c_{2} \Delta V_{D C, p u}\right), \\
& Q_{I C}^{*}=c_{3} \Delta V_{A C},
\end{aligned}
$$

where $c_{1,2,3}$ are well-tuned droop coefficient, $Q_{I C}^{*}$ and $P_{I C}^{*}$ denote the power exchange reference, and the PI gains are $K_{p}, K_{i}$. A positive $P_{I C}^{*}$ indicates that the IC has to transfer power from DC to AC grid, and vice versa if it is negative. The current reference $i_{o d}^{*}$ and $i_{o q}^{*}$ is derived from the resulting power exchange reference value:

$$
i_{d q}^{*}=i_{o d}^{*}+j i_{o q}^{*}=\frac{2}{3 V_{A C}}\left(P_{I C}^{*}-j Q_{I C}^{*}\right) .
$$

The resulting IC current reference will be used by the current controller to determine the input signal for the PWM block. The objective of this paper is to implement a current controller such that the current reference $i_{o d}^{*}$ and $i_{o q}^{*}$ are achieved. Conventionally, a PI controller is imposed because it is relatively simple as the IC system model is not required. However, determining PI gains for the IC can be a time consuming due to the varying operating points. In this paper, the first step to solve the control problem is studying the system model. Typically, an IC is interconnected with a resistor-inductor (RL) filter to deal with the current harmonics, as shown in Figure 3. By applying a Kirchoff's voltage law at the point of common coupling (PCC),

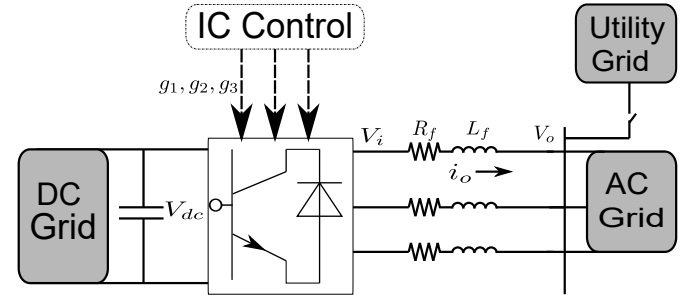

Figure 3. Topology of interlink converter (IC).

the three-phase voltage at $\mathrm{AC}$ side is given by

$$
v_{o_{a b c}}=v_{i_{a b c}}+R_{f} i_{a b c}+L_{f} \frac{d i_{a b c}}{d t} .
$$

Then, by assuming the switching signals $g_{1}, g_{2}, g_{3}$ bring the IC voltage $v_{i d, q}$ to its reference value $v_{i d, q}^{*}$ and the angular frequency $\omega$ is constant, the state space model is obtained and written in $d q$-frame:

$$
\begin{aligned}
& \dot{x}=A_{c} x+B_{c} u+E_{c} d, \\
& A_{c}=\left[\begin{array}{cc}
-\frac{R_{f}}{L_{f}} & \omega \\
-\omega & -\frac{R_{f}}{L_{f}}
\end{array}\right], B_{c}=\left[\begin{array}{cc}
\frac{1}{L_{f}} & 0 \\
0 & \frac{1}{L_{f}}
\end{array}\right], \\
& E_{c}=\left[\begin{array}{cc}
\frac{V_{D C}}{2 L_{f}} & 0 \\
0 & \frac{V_{D C}}{2 L_{f}}
\end{array}\right],
\end{aligned}
$$

where $x=\left[\begin{array}{ll}i_{o d} & i_{o q}\end{array}\right]^{T}$ is the state vector, $u=\left[\begin{array}{ll}v_{i d}^{*} & v_{i q}^{*}\end{array}\right]^{T}$ is the control variable and $d=\left[-v_{o d}-v_{o q}\right]^{T}$ is viewed as the disturbance variable. Suppose that $d$ is constant during the sampling period $T_{s}$, the model (3) can be discretized as

$$
x(k+1)=A x(k)+B u(k)+E d(k),
$$

where $A:=e^{A_{c} T_{s}}, B:=\int_{T_{s}}^{0} e^{A_{c} t} B_{c} d t, E:=\int_{T_{s}}^{0} e^{A_{c} t} E_{c} d t$ and $k$ denotes the $k$-th sampling time. This model will be used to describe the control problem which is discussed in next section.

\subsection{Problem Formulation}

The objective of IC current control is to regulate the IC current. To this end, the following function is minimized

$$
\left(i_{o d}^{*}(k)-i_{o d}(k)\right)^{2}+\left(i_{o q}^{*}(k)-i_{o q}(k)\right)^{2} .
$$

A MPC minimizes desired cost function over a finite horizon. By considering the system dynamic (3) and denoting the $\Delta u(k)$ as the increment of control input $u(k)=u(k-1)+\Delta u(k)$, for given measured state $x(0 \mid k)=x(k)$ and the previous con- 
trol input $u(0 \mid k)=u(k-1)$, the following cost function is considered to regulate the IC controller:

$$
\begin{aligned}
J= & \sum_{i=0}^{N-1}\left(x(i \mid k)-x(i \mid k)^{*}\right)^{T} Q\left(x(i \mid k)-x(i \mid k)^{*}\right) \\
& +\Delta u(i \mid k)^{T} R \Delta u(i \mid k),
\end{aligned}
$$

where $N$ is the prediction horizon, $x(i \mid k)$ and $u(i \mid k)$ denote the predicted state and control input, respectively. By assuming the predicted disturbance equals to the measured disturbance variable, the predicted state is given by:

$$
\begin{aligned}
& x(i \mid k)=A x(i-1 \mid k)+B u(i-1 \mid k)+E d(k), \\
& u(i \mid k)=u(i-1 \mid k)+\Delta u(k \mid k) .
\end{aligned}
$$

The matrices $Q$ and $R$ are the positive-definite weighting for the reference tracking error and increment control input, respectively. Moreover, to avoid over-modulation in the PWM block control, the input signal constraint is considered:

$$
u_{\min } \leq u(i \mid k) \leq u_{\max } .
$$

The state constraint is also taken into account to limit the current output

$$
x_{\min } \leq x(i \mid k) \leq x_{\max }
$$

Thus, the MPC problem can be written as follows:

$$
\min _{\Delta u(i \mid k), i=0, \ldots, N-1} J \text { in (4) }
$$

subject to (5), (6) (7), (8)

By transforming the problem into equivalent quadratic programming (QP) formulation, the problem can be solved using any standard nonlinear programming method [?]. Note that for a long prediction horizon, the problem (9) requires a huge computation load. In the next section, an Explicit MPC is discussed to reduce the computational burden of the implicit MPC.

\subsection{Explicit MPC}

The computational complexity to solve the MPC grows as the number of constraint and prediction horizon increase. The problem (9) consists of state and input constraints in which upper and lower bounds are needed since the IC provides bidirectional power exchange. This paper employs the Explicit MPC to solve the QP of the problem (9) with the reduced online computation load. This method minimizes the objective function offline, obtaining the state space subdivision into polyhedral regions in which the associated optimal control effort resides.

Substituting predicted control input (6) into (5) and denoting $u_{i}=u(i \mid k)$ and $x_{i}=x(i \mid k)$, the predicted state vector can be written as

$$
X(k)=\Theta x(k)+\Omega_{B} u(k-1)+\Omega_{E} d(k)+\Gamma \triangle U(k),
$$

where

$$
\begin{gathered}
\triangle U(k)=\left[\begin{array}{c}
\triangle u_{0} \\
\vdots \\
\triangle u_{N-2}
\end{array}\right], \\
\Gamma=\left[\begin{array}{ccc}
B & \ldots & 0 \\
\vdots & \ddots & \ldots \\
\sum_{i=1}^{N-2} A^{i} B & \ldots & B
\end{array}\right],
\end{gathered}
$$

and

$$
\begin{gathered}
X(k)=\left[\begin{array}{c}
x_{1} \\
\vdots \\
x_{N-1}
\end{array}\right], \Omega_{B}=\left[\begin{array}{c}
B \\
\vdots \\
\sum_{i=0}^{N-2} A^{i} B
\end{array}\right], \\
\Theta=\left[\begin{array}{c}
A \\
\vdots \\
\vdots \\
A^{N-1}
\end{array}\right], \Omega_{E}=\left[\begin{array}{c} 
\\
\sum_{i=0}^{N-2} A^{i} E
\end{array}\right] .
\end{gathered}
$$

In this paper, it is assumed that the predicted state references equal to the state referece at time sampling $k, x_{i}^{*}=x^{*}(k)$ for $i=1, \ldots, N-1$. Thus, problem (4) can be rewritten into a QP problem:

$$
\min _{\Delta U(k)} \Delta U^{T}(k) H \Delta U(k)+\theta(k)^{T} F \Delta U(k)
$$

subject to

$$
\begin{aligned}
& A_{u} \Delta U(k) \leq B_{u}\left(u_{\max }-u(k-1)\right) \\
& A_{x} \Delta U(k) \leq B_{x}\left(x_{\max }-x_{\min }\right)+W \theta(k),
\end{aligned}
$$

where $\theta(k)=\left[x(k)^{T} u(k-1)^{T} d(k)^{T} x_{1}^{*}(k)^{T} \ldots x_{N-1}^{*}(k)^{T}\right]$ and $H, F, A_{u}, A_{x}, B_{u}, B_{x}, W$ are the appropriate matrices such that (9) and (11) are quasi-equivalent. The Explicit MPC solves the problem using the multi-parametric programming and obtains the optimal decision as a piecewise affine function 


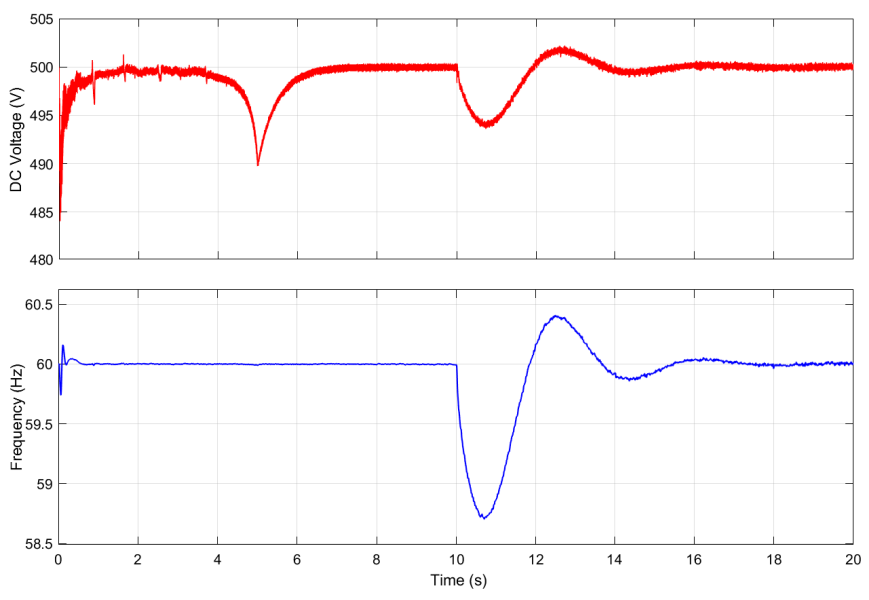

Figure 4. DC voltage and AC frequency responses for the first case. The DC voltage deviates at $t=3 \mathrm{~s}$ but is restored at $t=7 \mathrm{~s}$. The utility is disconnected at $t=10 \mathrm{~s}$, resulting frequency and DC voltage deviations for 6 seconds.

(PWA) [?]:

$$
\Delta U(k)=\left\{\begin{array}{ccccc}
K_{1} \theta(k)+c_{1} & \text { if } & \theta(k) & \in \mathcal{R}^{1} \\
\vdots & & & & \vdots \\
K_{q} \theta(k)+c_{q} & \text { if } & \theta(k) & \in \mathcal{R}^{q}
\end{array}\right.
$$

where $\mathcal{R}^{j}$ for $j=1, \ldots, q$ are the resulting subsets in which the control law $K_{j} \theta(k)+c_{j}$ is valid when $\theta(k) \in \mathcal{R}^{j}$. Then, the input signal $u(k)=u(k-1)+\Delta u_{0}$ is applied to the system where $\Delta u_{0}$ is the first two component of the vector $\Delta U(k)$. Note that the optimal control input (12) is in a lookup table form. Thus, the online computation is reduced since the optimal solution is solved offline.

\section{Simulation Result}

In this section, the simulation result of the Explicit MPCbased IC current control is discussed. The explicit optimal input signal is obtained by using the multi-parametric toolbox (MPT) in MATLAB with the parameters described in Table 1 Then, it is applied to regulate the IC current of hybrid AC/DC microgrid. The system parameters are taken from [3]. Two simulation cases are employed to show the performance of the proposed method.

In the first case, the utility grid is connected at time $t \in[1,10]$ s. Suppose that the AC sub-grid has $65 \mathrm{~kW}$ power source and 20 kW load, while DC microgrid experiences power source-load imbalance at time $t=4 \mathrm{~s}$. Figure 4 shows the DC voltage and $\mathrm{AC}$ frequency responses for this simulation case. The power im-

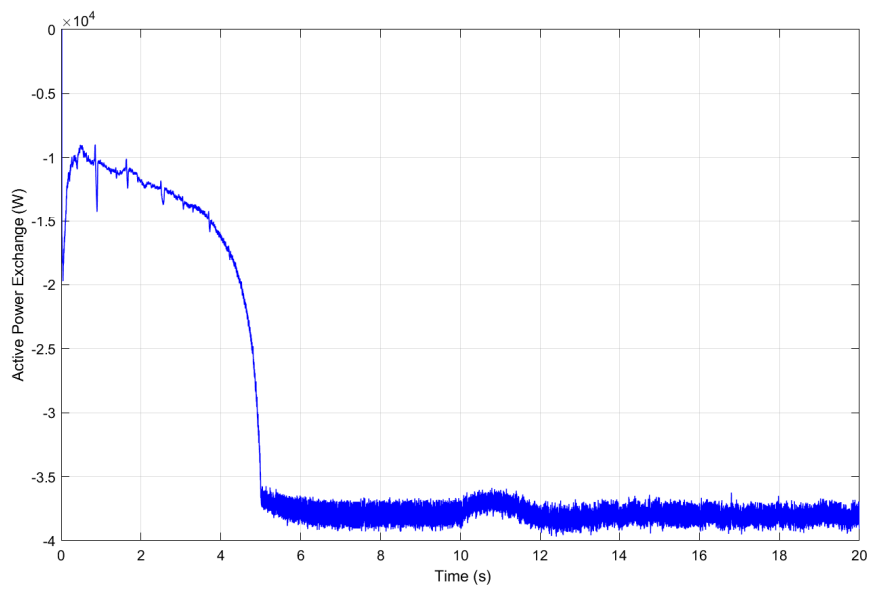

Figure 5. Active power exchange for the first case. Around $3.8 \mathrm{~kW}$ active power is transmitted from $\mathrm{AC}$ to $\mathrm{DC}$ sub-grid in order to restore the DC voltage to its nominal value.

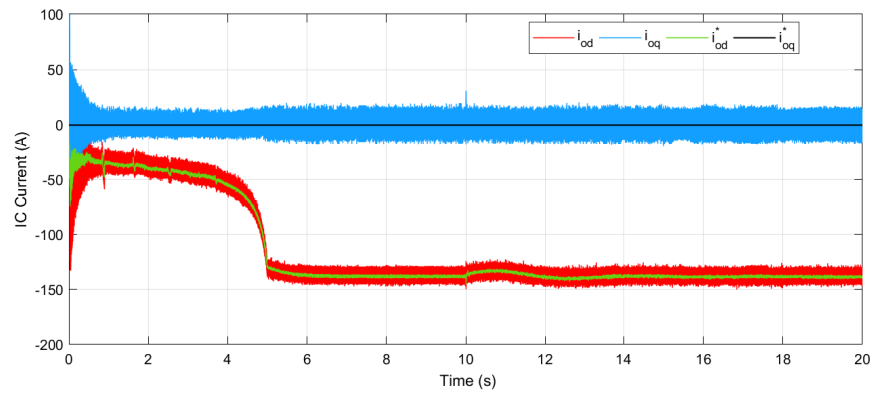

Figure 6. IC current output for the first case: reference tracking is achieved.

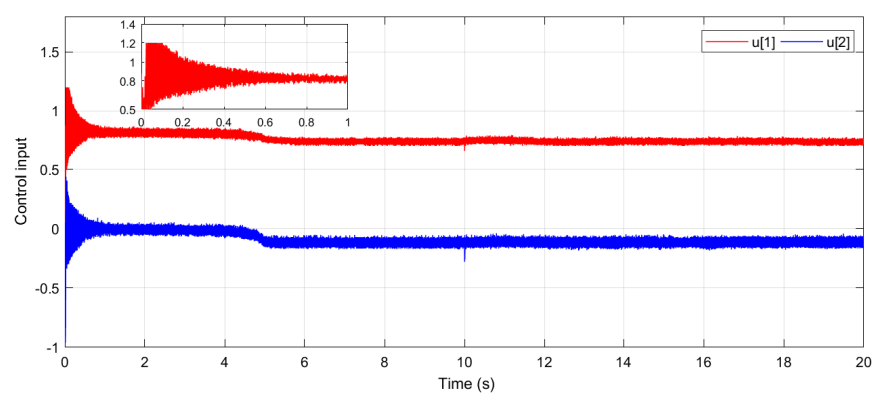

Figure 7. Control input for the first case. The control input is constrained in its maximum value 1.2 at $t \in[0.01,0.12]$.

balance happens at $t=3 \mathrm{~s}$. As a result, the DC voltage deviates to $490 \mathrm{~V}$. The IC controller successfully restores the DC voltage to the nominal value $500 \mathrm{~V}$ at $t=7 \mathrm{~s}$. Besides, the frequency is maintained in $60 \mathrm{~Hz}$ when the microgrid is in grid-connected mode. However, when the utility grid is disconnected from the microgrid, the frequency deviates to $58.4 \mathrm{~Hz}$. Since AC subgrid has a sufficient power source, the frequency can be brought 


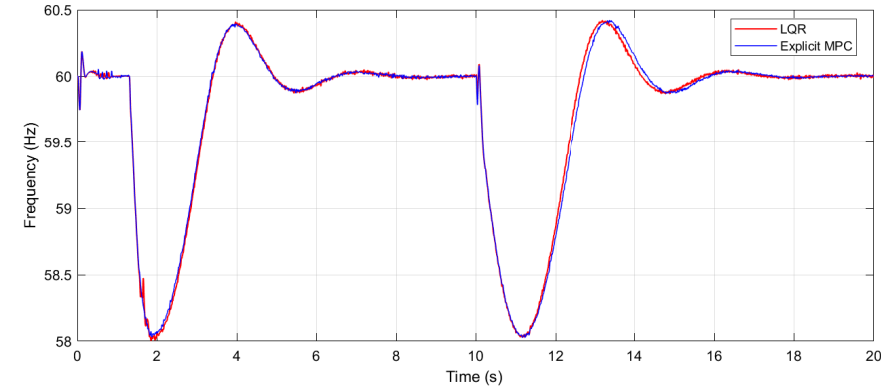

Figure 8. Frequency response for the second case: both controllers show comparable results.

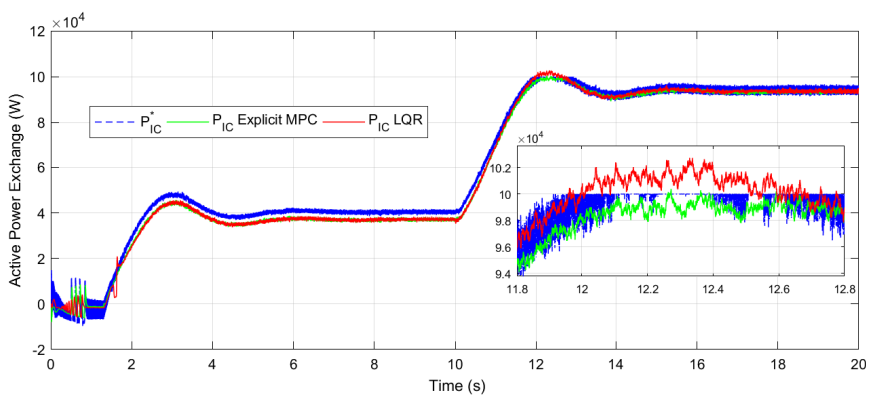

Figure 9. Active power exchange under high loading condition in $\mathrm{AC}$ sub-grid. The exchanged power of LQR exceeds the maximum value while the Explicit MPC limits the power exchange in its maximum value at $t \in[12,16]$.

back to $60 \mathrm{~Hz}$. Figure 5 shows the active power exchange has negative value, which means the active power flows from $\mathrm{AC}$ to DC sub-grid. In addition, the current output and control signal responses are shown in Figures 6 and 7 respectively. Figure 6 shows that the proposed current control steers the current output to the reference value while maintaining the input signal in the acceptable range (Figure 7 ).

In the second case, the AC sub-grid experiences high loading condition in the stand-alone mode. At first, utility grid is connected to the microgrid for $1.3 \mathrm{~s}$ before it is detached. Furthermore, at $t=10 \mathrm{~s}$, the AC load increases from $84 \mathrm{~kW}$ to $126 \mathrm{~kW}$. Meanwhile, the battery in DC sub-grid is assumed to have state of charge (SOC) of $60 \%$ which is enough for the DC sub-grid. In this case, the performance of proposed current control is compared to the existing model-based current control: LQR [6]. Figure 8] shows the frequency response of Explicit MPC and LQR control methods. Both controllers maintain the frequency both in the transient and steady state.

The current constraints are taken into account with the value defined in Table 1. This value is obtained by assuming that the IC has an acceptable maximum power of $100 \mathrm{~kW}$. Figure 9 demonstrates the resulting active power exchange. The positive

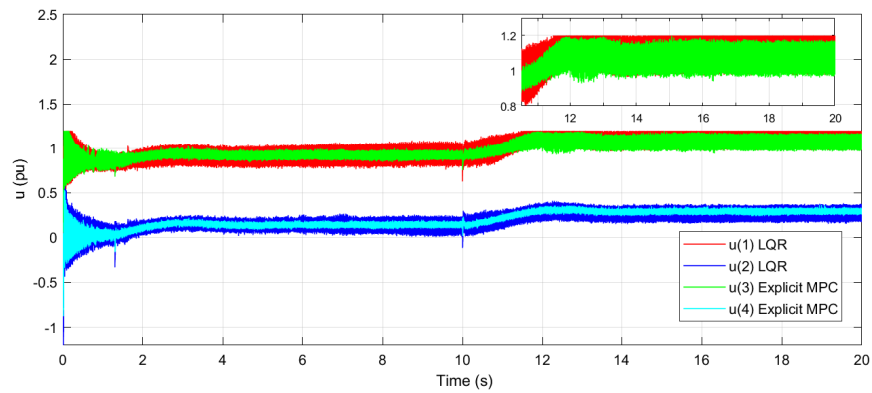

Figure 10. Control input for the second case: the Explicit MPC lessens the control input of the LQR method.

Table 1. Explicit MPC parameter

\begin{tabular}{cc}
\hline Parameter & Value \\
\hline$Q$ & $0.5 I$ \\
$R$ & $120 I$ \\
$N$ & 7 \\
$u_{\max }$ & {$[1.2 ; 1.2]$} \\
$x_{\min }$ & {$[-260 ;-30]$} \\
\hline
\end{tabular}

value represents the power exchange flows from DC to AC sub-grid. As can be seen in Figure 9, when microgrid is in maximum load condition, the power exchange using LQR method goes around $120 \mathrm{~kW}$, which is larger than the maximum value. Meanwhile, the Explicit MPC can maintain the power exchange in the acceptable value. Furthermore, in view of optimality, the Explicit MPC yields less control input variation compared to the LQR method as can be seen in Figure 10.

\section{Conclusions}

In this paper, an optimal control method is studied for the interlink converter controller to regulate the inverter current output. The MPC method is used to achieve current reference tracking with optimal control effort. In addition, the method gives an advantage as it allows the controller to include input and state constraints. Thereby, the safety of component can be guaranteed. Furthermore, an Explicit MPC is employed to avoid the controller solves the optimization problem at each sampling time. Hence, the proposed controller is more suitable for practical use than the conventional MPC as the online computation load is reduced. A computer simulation of a hybrid AC/DC microgrid is employed to show the performance of the proposed controller. 


\section{Conflict of Interest}

No potential conflict of interest relevant to this article was reported.

\section{Acknowledgement}

This study was supported by the Research Program funded by the Seoul National University of Science and Technology (SeoulTech).

\section{References}

[1] P. C. Loh, D. Li, Y. K. Chai, and F. Blaabjerg, "Autonomous operation of hybrid microgrid with $\mathrm{AC}$ and DC subgrids," IEEE Transactions on Power Electronics, vol. 28, no. 5, pp. 2214-2223, 2013. https://doi.org/10. 1109/TPEL.2012.2214792

[2] N. Eghtedarpour and E. Farjah, "Power control and management in a hybrid AC/DC microgrid," IEEE Transactions on Smart Grid, vol. 5, no. 3, pp. 1494-1505, 2014. https://doi.org/10.1109/TSG.2013.2294275

[3] I. Fitri, J. S. Kim, and H. Song, "A robust suboptimal current control of an Interlink converter for a hybrid AC/DC microgrid," Energies, vol. 11, no. 6, article no. 1382, 2018. https://doi.org/10.3390/en11061382

[4] E. M. Suhara, M. Nandakumar, and K. Mathew, "Novel adaptive hysteresis current control of bidirectional three phase PWM converter under reduced switching scheme," in Proceedings of IEEE International Conference on Power Electronics, Drives and Energy Systems (PEDES), Trivandrum, India, 2016. https://doi.org/10.1109/PEDES. 2016.7914452

[5] N. Jin, S. Hu, C. Gan, and Z. Ling, "Finite states model predictive control for fault-tolerant operation of a threephase bidirectional AC/DC converter under unbalanced grid voltages," IEEE Transactions on Industrial Electronics, vol. 65, no. 1, pp. 819-829, 2018. https://doi.org/10. 1109/TIE.2017.2686342

[6] D. R. Aryani, J. S. Kim, and H. Song, "Interlink converter with linear quadratic regulator based current control for hybrid AC/DC microgrid," Energies, vol. 10, no. 11, article no. 1799, 2017. https://doi.org/10.3390/en10111799
[7] M. P. Akter, S. Mekhilef, N. M. L. Tan, and H. Akagi, "Model predictive control of bidirectional AC-DC converter for energy storage system," Journal of Electrical Engineering and Technology, vol. 10, no. 1, pp. 165-175, 2015.

[8] J. Zhang, D. Guo, F. Wang, Y. Zuo, and H. Zhang, "Control strategy of interlinking converter in hybrid AC/DC microgrid," in Proceedings of International Conference on Renewable Energy Research and Applications (ICRERA), Madrid, Spain, 2013, pp. 97-102.https://doi.org/10.1109/ ICRERA.2013.6749733

[9] D. R. Aryani, J. S. Kim, and H. Song, "Suppression of PV output fluctuation using a battery energy storage system with model predictive control," International Journal of Fuzzy Logic and Intelligent Systems, vol. 17, no. 3, pp. 202-209, 2017. https://doi.org/10.5391/IJFIS.2017.17.3 202

[10] S. J. Kim and J. M. Lee, "Optimal design of the PID controller using a predictive control method," International Journal of Fuzzy Logic and Intelligent Systems, vol. 5, no. 1, pp. 69-75, 2005.

[11] J. B. Rawlings and D. Q. Mayne, Model Predictive Control Theory and Design. Madison, WI: Nob Hill Publishing, 2009.

[12] A. Bemporad, M. Morari, V. Dua, and E. N. Pistikopoulos, "The explicit linear quadratic regulator for constrained systems," Automatica, vol. 38, no. 1, pp. 3-20, 2002. https: //doi.org/10.1016/S0005-1098(01)00174-1

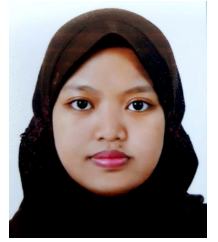

Ismi Rosyiana Fitri is a Ph.D. candidate at the Department of Electrical and Information Engineering, Seoul National University of Science and Technology (SeoulTech). She has been working on research related to optimal control, distributed control, and its application to power systems.

E-mail: ismirosyiana@gmail.com

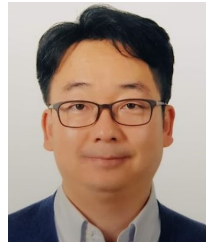

Jung-Su Kim received his B.S., M.S. and Ph.D. degrees in Electrical Engineering from Korea University. He was a post-doc at CDSL, Seoul National University, in 2005, at IST, University of Stuttgart, Germany from 2006-2007, and Systems Biology Laboratory, University of Leicester, UK, 
in 2008. Since 2009, he has been with the Department of Electrical and Information Engineering, SeoulTech. His research interests include MPC, distributed control, Microgrid, and deep learning based robot path planning.

E-mail: jungsu@ seoultech.ac.kr 